\title{
Complexity and line of critical points in a short-range spin-glass model
}

\author{
M. Campellone \\ Dipartimento di Fisica and Sezione INFN, Università di Roma "La Sapienza" \\ Piazzale A. Moro 2, 00185 Rome (Italy) \\ e-mail: matteo.campellone@roma1.infn.it \\ F. Ritort \\ Departament de Fisica Fonamental, Facultat de Fisica \\ Universitat de Barcelona, Diagonal $64^{7}$ \\ 08028 Barcelona (Spain). \\ E-Mail: ritort@ffn.ub.es
}

(November 19, 2018)

\begin{abstract}
We investigate the critical behavior of a three-dimensional short-range spin glass model in the presence of an external field $\epsilon$ conjugated to the Edwards-Anderson order parameter. In the meanfield approximation this model is described by the Adam-Gibbs-DiMarzio approach for the glass transition. By Monte Carlo numerical simulations we find indications for the existence of a line of critical points in the plane $(\epsilon, T)$ which separates two paramagnetic phases and terminates in a critical endpoint. This line of critical points appears due to the large degeneracy of metastable states present in the system (configurational entropy) and is reminiscent of the first-order phase transition present in the mean-field limit. We propose a scenario for the spin-glass transition at $\epsilon=0$, driven by a spinodal point present above $T_{c}$, which induces strong metastability through Griffiths singularities effects and induces the absence of a two-step shape relaxation curve characteristic of glasses.
\end{abstract}

Among all different approaches to understand the glass transition the thermodynamic theory of Adam-Gibbs-Di Marzio (AGM) has deserved a lot of interest during the last decades [1]. The AGM theory predicts the occurrence, at a Kauzmann temperature $T_{K}$, of a second order phase transition for the metastable undercooled liquid where the configurational entropy (also called complexity) vanishes. The validity of the AGM theory for real glasses has never been demonstrated so the correct description of the glass transition still remains open [2]. An alternative dynamical approach was proposed in the eighties to describe relaxational processes in the undercooled liquid regime, experimentally observed in scattering and dielectric measurements. This received the name of mode-coupling theory (MCT) [3].

Quite recently it has been realized that spin glasses are models which account for both the thermodynamic (AGM) and the dynamical (MCT) approaches [4]. Although spin glasses are models with quenched disorder (and structural glasses are not) this is not an essential difference because the existence of a crystal phase in structural glasses has no dramatic effect in the dynamics of the (disordered) metastable undercooled liquid phase. Unfortunately, up to now this connection between spinglasses and glasses remains only at the mean-field level and it is not clear what happens if one considers shortrange interactions. In fact, concepts such as complexity in AGM or the ergodicity parameter in ideal MCT are originally mean-field and it is not clear what is their relevance in short-ranged realistic systems.

Recently it has been suggested [6,5] that the effect of the complexity could be observed through numerical sim- ulations in a generic glassy system coupling two replicas by introducing a term in the Hamiltonian of the type $-\epsilon q$ ( $\epsilon$ being the conjugate field of the order parameter $q$ which is the overlap between the configurations of the two replicas [7]). Through the study of an exactly solvable spin-glass model it has been shown the existence of a first order transition line $T_{c}(\epsilon)$ with a critical end-point [5]. Their result is an explicit check of the fact that the glass transition for $\epsilon=0$ (where the complexity vanishes) is a first order phase-transition (in the sense that the order parameter $q$ is discontinuous) and the point $T_{c}=T_{c}(\epsilon=0)$ is a tricritical point. Again, this result has been obtained within the mean-field approximation and it is unclear to what extent this result is valid in a finite-dimensional model. Recent numerical simulations on a short-range version of $p$-spin Ising spin glass [8, 9] have shown that the mean-field discontinuous transition becomes continuous in finite dimensions. So the characteristic first-order transition predicted within mean-field theory dramatically changes in finite dimensions. In this work we want to show that this is not completely true and that some features of the mean-field approximation indeed survive in finite dimensions although they must be appropriately interpreted. This allows to interpret our findings in terms of a picture for a continuous spin-glass transition induced by the strong metastability and driven by the collapse of the complexity or configurational entropy. Furthermore, our results point in the direction that disordered systems in three dimensions are very well described by a line of critical points (characteristic of systems at their lower critical dimension) in agreement with recent numerical simulations in the Edwards-Anderson 
model in three dimensions [10].

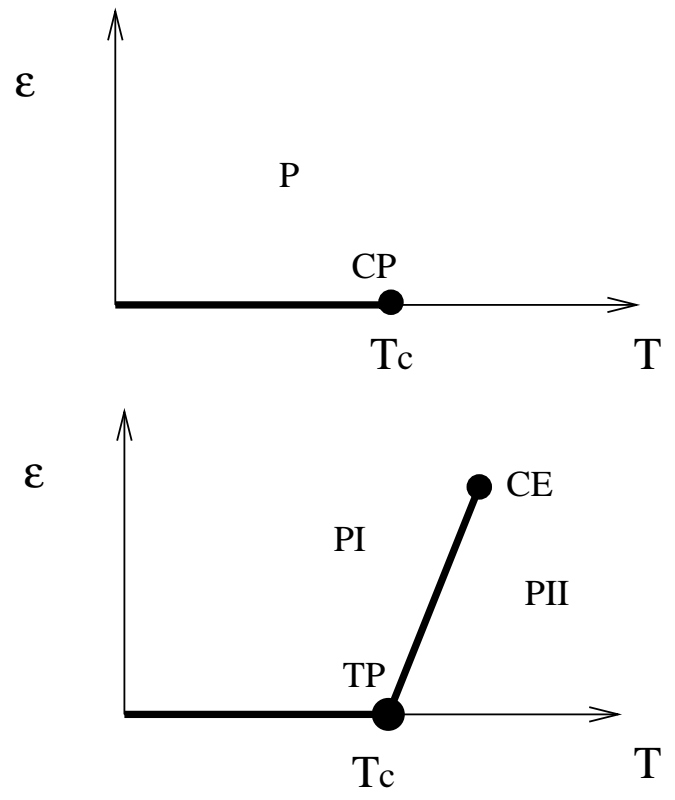

FIG. 1. Different phases for the Edwards-Anderson model (above) and the short-range p-spin model (below). In the former case there is a single paramagnetic phase, in the latter two different paramagnetic phases divided by a critical line which connects a tricritical point (TCP) and a critical endpoint (CE) (for $p$ even the line $T P-C E$ exists also for $\epsilon<0$ ). In both cases the spin-glass phase is present only for $\epsilon=0$.

We shall consider a recently introduced short-range $p$ spin glass model [8.9] which is defined on a $d$ dimensional hypercubic lattice. On each site of the lattice there are $M$ spins interacting with the following Hamiltonian

$$
\mathcal{H}_{p}(\{\sigma\})=-\sum_{<i_{1}, \cdots, i_{p}>l_{1}, \cdots, l_{p}=1}^{L^{d}} \sum_{i_{1} \ldots i_{p}}^{M} \sigma_{i_{1}}^{l_{1} \ldots l_{p}} \cdots \sigma_{i_{p}}{ }^{l_{p}} .
$$

By $\sum_{<i_{1}, \cdots, i_{p}>}^{L^{d}}$ we sum over all sites of the lattice all possible groups of $p$ spins that can be formed between spins on the same site and spins of adjacent sites. In this work we consider Ising spin variables. By ${\sigma_{i}}_{i_{r}}{ }^{{ }_{r}}$ we denote the $l_{r}^{\text {th }}$ spin of site $i_{r}$ with the index $l_{r}$ running from 1 to $M$. For $p=2$ (11) corresponds to the Edwards-Anderson model. Although this model may seem quite artificial it has the advantage of including multispin interactions in a lattice without introducing new symmetries which may change the degeneracy of the ground state [11].

Here we consider two identical coupled models (each defined through (11)) via the following Hamiltonian

$$
\mathcal{H}_{p}^{2}(\{\sigma\},\{\tau\})=\mathcal{H}_{p}(\{\sigma\})+\mathcal{H}_{p}(\{\tau\})-\epsilon V q
$$

where $V=L^{3}$ is the volume and $V q=\sum_{i=1}^{V} \sigma_{i} \tau_{i}$ defines the order parameter. Because the $\epsilon=0$ transition in this model is continuous we naively expect that (similarly as it happens in the Edwards-Anderson model) there is no phase transition for $\epsilon \neq 0$. In mean-field models with a one-step replica broken phase, a transition exists for finite $\epsilon$ [5]. In that case, there is a transition line which separates two paramagnetic phases with a finite latent heat (which vanishes at the critical endpoint). The two possible phases are depicted in figure 1. The EdwardsAnderson order parameter $q_{E A}$ (defined as the infinitetime limit of the equilibrium autocorrelation function [7]) displays a finite jump across the line $T_{c}(\epsilon)$ which vanishes at the critical endpoint. Here we find strong indications, through Monte Carlo simulations, that this first-order line $T_{c}(\epsilon)$ persists in finite dimensions but becomes a line of critical points. So, in finite dimensions the first-order line becomes continuous (i.e. $q_{E A}$ is continuous when crossing the transition line and there is no latent heat), the critical endpoint displaying a higher-order singularity.

In this paper we will focus our research of (11) for case $M=3, p=4$ in $D=3$. Measurements of the spin-glass susceptibility for (11) show that this model has a continuous finite-temperature phase transition at $T_{c} \simeq 2.6$ with a divergent spin-glass susceptibility and a small negative specific heat exponent [8]. To evidenciate a phase transition for finite $\epsilon$ which separates two different paramagnetic phases we have done a detailed Monte Carlo study [12] of the Binder parameter as a function of both the coupling $\epsilon$ and the temperature in the paramagnetic region. The Binder parameter is usually defined through the relation,

$$
g(\epsilon, T)=\frac{1}{2}\left(3-\frac{\overline{(q-<q>)^{4}}}{\left(\overline{(q-<q>)^{2}}\right)^{2}}\right)
$$

where $<. .>$ stands for statistical average and $\overline{(.)}$ for disorder average. Concerning the Binder parameter we expect that it should vanish everywhere in both paramagnetic phases except at the critical line where it should be finite. So, if we fix $\epsilon$ (or, equivalently the temperature) and vary the temperature (equivalently $\epsilon$ ) we expect the presence a maximum approximately located on the transition line at a temperature $T_{c}(L, \epsilon)$. The results for $g(\epsilon, T)$ are shown in figure 2 2 for $T=3.2$ and different sizes. Note that the Binder parameter shows a maximum located at $\epsilon=0.05$. As a comparison we have done simulations for the Edwards-Anderson model in three dimensions above $T_{c}$ by coupling two replicas which evidenciate the absence of a maximum in this case. So the presence of a maximum in $g(\epsilon, T)$ already for small sizes is a main feature of this model. For $L=3,4,5$ finite-size corrections appear to be quite strong (this was already observed in [8] by measuring the $P(q)$ ) and the position of the maximum of the Binder parameter as well as its value both shift with $L$. Nevertheless, the maximum of the Binder parameter for $L=6$ superimposes with the maximum for $L=5$ and the Binder parameter goes to 
zero outside from the maximum as $L$ increases. This result indicates the presence of a critical line which separates two paramagnetic phases. A more stringent test of this result requires simulating larger sizes than those we did. Unfortunately for $L=7$ the thermalization time is much larger than what we can afford with the present numerical methods. Actually, a test of the needed thermalization time shows that it grows dramatically with $L$ and for $L=7$ and $T=3.2$ this may be larger than several hundreds of million of Msteps for a non vanishing fraction of disorder realizations. As we will argue later this is consequence of the strong metastability and the highly corrugated landscape characteristic of this model which, on the other hand, induces the existence of this critical line (actually thermalization is much easier in the Edwards-Anderson model). Note that the fact that the maximum value of $g$ along the critical line is smaller than one (approximately 0.2 ) is an indication that the transition is continuous in $q$ (if there were a finite jump in $q$ the maximum value of $g$ should be 1). Moreover, our data do not show any indication of a jump in the value $q(\epsilon)$ as a function of the temperature (or $q(T)$ as a function of $\epsilon$ ) in the region of the maximum of $g$.

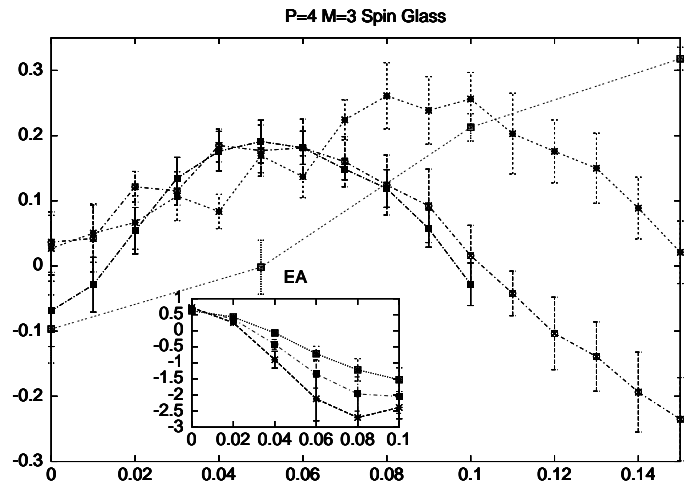

FIG. 2. Binder parameter as a function of $\epsilon$ at temperature $T=3.2$ for different sizes $(L=3$ empty squares, $L=4$ filled circles, $L=5$ empty circles, $L=6$ filled squares). The same plot for the Edwards-Anderson model (inset) with $T=1.3,1.4,1.5\left(T_{c} \simeq 1.2\right.$ 13] $)$ shows a completely different behavior compared to the $p=4$ model.

In what follows we try to estimate the shape of this critical line using finite-size scaling techniques. A detailed investigation of the critical line as well as its critical exponents is presently out of reach due to the smallness of the sizes studied. Still we can approximately determine its shape. Let us suppose (as data of figure 2 suggests) that there is a critical line $\epsilon \sim C\left(T-T_{c}\right)^{\lambda}$ where $C, \lambda>0$ and $T_{c} \simeq 2.60$. Assuming the validity of the scaling hypothesis we may write $g(T, \epsilon) \equiv \hat{g}\left(\epsilon\left(T-T_{c}\right)^{-\lambda}\right)$. In figure 3 we plot the scaling behavior within the scaling region for different values of $T$ and $\epsilon$ for the largest size $L=6$. The scaling is quite good and proves two re- sults: 1) The position of the maximum stays along a well defined line in the $(\epsilon, T)$ plane and 2) The value of the maximum of $g$ is the same everywhere along that line. A good collapse of data is obtained with an exponent $\lambda \simeq 2$. The position of the maximum of the scaled data yields a value of $C \simeq 0.17$ and $g_{\max } \simeq 0.2$. This value is universal along the critical line and approximately coincides with the value of the Binder parameter at the tricritical point [8]. Although we expect that the critical line will have some $L$ dependence, very similar results obtained for $L=5$ indicate that our estimate of the critical line for $L=6$ is a good estimate of the true (infinite volume) line.

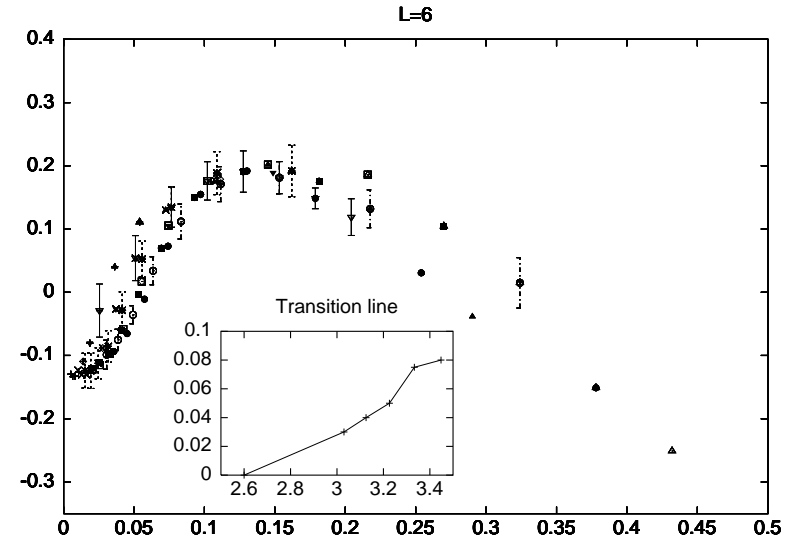

FIG. 3. Binder parameter plotted as a function of $\epsilon(T-2.6)^{-2}$ in the range $.01 \leq \epsilon \leq .09,3.0 \leq T \leq 4.0$ for $L=6$. In the inset we show the estimated critical line (see the text) compared to the points for $L=5,6$ where appears a maximum of $g$ in the plane $(\epsilon, T)$.

Now we discuss the physical interpretation of this critical line. As already said in the introduction, the presence of this transition line is consequence of the existence of an exponentially large number (with the volume $\exp (\alpha V), \alpha$ being a coefficient) of metastable states in this model. In mean-field theory (AGM or ideal MCT) metastable states have an infinite lifetime so there are infinitely large barriers which separate them. This is the reason why in mean-field theory ergodicity already breaks at the mode-coupling (also called dynamical) temperature. In short-ranged systems or real glasses metastable states decay by nucleation processes so ergodicity is always restored. A typical feature of the ideal MCT singularity is the characteristic two-step relaxational decay in correlation functions, the so-called $\alpha$ and $\beta$ processes. In ideal MCT the typical relaxation time associated to the $\alpha$ process diverges at $T_{d}$ and the ergodicity parameter jumps discontinuously at $T_{d}$. An accurate study of correlation functions reveals that the two-step characteristic relaxation curve is absent in the present model (11) [8,9]. The absence of a plateau in these curves indicate that do not exist two well separated time-scales $(\alpha$ and $\beta$ ), like in generic glass-forming liquids, but a con- 
tinuous hierarchy of time scales for nucleation processes. We interpret this result as consequence of the continuous nature of the transition everywhere in the critical line $\epsilon-T$. A possible scenario for the potential function for this type of transition is depicted in figure 1 . There is not a typical time scale for nucleation process (where a small excited droplet or bubble decays from $q=q_{E A}$ to $q=0)$ and the potential around the secondary minimum $q=q_{E A}$ is marginally stable. Across the critical line the Edwards-Anderson parameter $q_{E A}$ is continuous but $\frac{d q_{E A}}{d \epsilon}$ is discontinuous, the potential being completely flat in $q$. Note that at $T=T_{c}, \epsilon=0$ where the complexity vanishes, the Edwards-Anderson parameter is also continuous in agreement with the absence of the two-step relaxation in this model. The complexity in this model is defined by the height of the secondary saddle point which moves towards $q=0$ for $T=T_{c}$. The present scenario is very different to that found in the Edwards-Anderson model in finite dimensions where no additional spinodal point (apart from the paramagnetic one $q=0$ ) is found above $T_{c}$. Concerning dynamical processes and thermalization effects this model behaves also quite differently. Spatial regions may be frozen if their local temperature (measured always respect to the intensity of the local interaction) is low enough for nucleation processes to decay very slowly. This is apparent from figure 1 where the secondary saddle point may become a stable minima in certain Griffiths regions. This effect has been observed in numerical studies of the $P(q)$ where a secondary peak in that distribution function has been observed already for small sizes for certain disorder realizations [8].

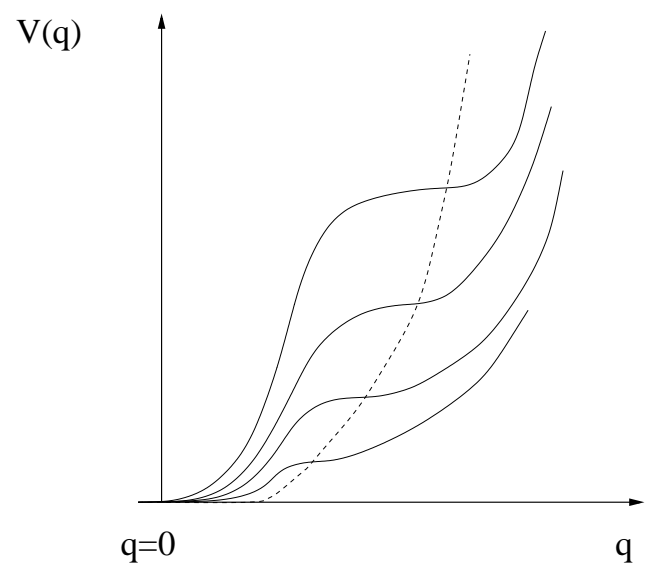

FIG. 4. Potential function for the short-range p-spin spin glass at $\epsilon=0$ above $T_{c}$ as a function of temperature (from high to low temperatures from above to below). The secondary minimum at $q \neq 0$ is a spinodal point. The height of that secondary saddle point is the complexity (the logarithm of the number of metastable states per site) which vanishes at $T_{c}$. The potential at $T=T_{c}$ becomes flat and $q_{E A}$ vanishes at $T_{c}$ (following the dashed line).

In summary, we have studied a short-range spin-glass model which in the mean-field approximation is well consistent with the Adam-Gibbs-DiMarzio theory and with the ideal mode-coupling theory. In the $\epsilon-T$ plane we have found evidence for a line of critical points. The sole existence of this transition suggests that a bunch of metastable states do change the high-temperature behavior through their configurational entropy or complexity. The interest of this study is that the full analysis is done in the high-temperature phase where thermalization is easier to achieve (but still, huge thermalization times are needed for large sizes) compared to the lowtemperature region. To our knowledge, such an analysis of this paramagnetic-paramagnetic transition has not been previously done for spin glasses. The main caveat of our results is that we cannot exclude the possibility that we are observing a crossover behavior where the maximum value of $g$ would eventually vanish for $L \rightarrow \infty$ [14. Unfortunately, metastability slows very much thermalization even in the high-temperature region. Further studies should extend the present analysis to the study of the potential energy landscape and a numerical estimate of the configurational entropy for this model (such as has been done for glass forming liquids 15]) as well as the role of the Griffiths singularities in the dynamics. Finally, because a finite $\epsilon$ breaks the $q \rightarrow-q$ symmetry the existence of this critical line cannot be simply explained in terms of a low-temperature spin-glass phase characterized by only two symmetrically related equilibrium states. So probably one must invoke more complex descriptions for the ground state structure and the type of excitations in the spin-glass phase.

Acknowledgments. We thank G. Parisi for discussions and F. G. Padilla for a careful reading of the manuscript. This work has been supported by the Ministerio de Educación y Ciencia in Spain through the project PB97-0971. M.C. thanks Fondazione Angelo Della Riccia for financial support.

[1] J. H. Gibbs and E. A. Di Marzio, J. Chem. Phys. 28373 (1958); G. Adams and J. H. Gibbs, J. Chem. Phys. 43 139 (1965)

[2] C. A. Angell, Science, 267, 1924 (1995)

[3] For review see, W. Gotze, Liquid, freezing and the Glass transition, Les Houches (1989), J. P. Hansen, D. Levesque, J. Zinn-Justin editors, North Holland

[4] T. R. Kirkpatrick and D. Thirumalai, Phys. Rev. B 36, 5388 (1987); T. R. Kirkpatrick and P. G. Wolyness, Phys. Rev. B 36, 8552 (1987).

[5] S. Franz, G. Parisi, Phys. Rev. Lett. 79, 2486 (1997), Physica A 261, 317 (1998)

[6] M. Cardenas, S. Franz and G. Parisi, J. Phys. A 31, L163 
(1998); J. Chem. Phys. 110, 1726 (1999)

[7] For introductory texts, see M. Mézard, G. Parisi and M. A. Virasoro, "Spin Glass Theory and Beyond" World Scientific, (1987); K. Binder and A. P. Young, Rev. Mod. Phys. 58 (1986) 801; K. H. Fischer and J. A. Hertz, "Spin Glasses" Cambridge University Press, (1991).

[8] M. Campellone, B. Coluzzi and G. Parisi, Phys. Rev. B 58, 12081 (1998); M. Campellone, G. Parisi and P. Ranieri, Phys. Rev. B 59, 1036; S. Franz and G. Parisi, Critical properties of a three dimensional p-spin model, Preprint cond-mat 9805088.

[9] E. Marinari, C. Naitza, G. Parisi, M. Picco, F. Ritort and F. Zuliani, Phys. Rev. Lett. 811698 (1998); G. Parisi, M. Picco and F. Ritort, Phys. Rev. E 60, 58 (1999).

[10] S. Caracciolo and M. Palassini, Phys. Rev. Lett. 82, 5128 (1999)

[11] D. Alvarez, S. Franz and F. Ritort, Phys. Rev. B 54, 9756 (1996) and references therein

[12] Monte Carlo simulations use the parallel tempering algorithm with 14 temperatures in the temperature range $3.0-5$. We simulated four different lattice sizes $L=3,4,5,6$ with $100,100,100,50$ samples and $2^{14}, 2^{17}, 2^{19}, 2^{20}$ thermalization steps respectively. Statistics was collected in a time window four times the previous thermalization times.

[13] E. Marinari, G. Parisi and J. J. Ruiz-Lorenzo in Spin Glasses and Random Fields Ed. by A. P. Young, (World Scientific, Singapore, 1998)

[14] Such strong finite-size effects have been observed in the disordered hierarchichal lattice, M. A. Moore, H. Bokil and B. Drossel, Phys. Rev. Lett. 81, 4252 (1998).

[15] F. Sciortino, W. Kob and P. Tartaglia, Preprint condmat 9906081

[16] A. J. Bray and M. A. Moore in Heidelberg Colloquium in Glassy dynamics, edited by J. L. Van Hemmen and I. Morgenstern, Lecture Notes in Physics Vol. 275 (Springer-Verlag, Heidelberg, 1987) 